\title{
Fast-ion orbit sensitivity of neutron emission spectroscopy diagnostics
}

Järleblad, H.; Stagner, L.; Salewski, M.; Eriksson, J.; Benjamin, S.; Madsen, B.; Nocente, M.; Rasmussen, J.; Schmidt, B.S.

Published in:

Review of Scientific Instruments

Link to article, DOI:

$10.1063 / 5.0040696$

Publication date:

2021

Document Version

Peer reviewed version

Link back to DTU Orbit

Citation (APA):

Järleblad, H., Stagner, L., Salewski, M., Eriksson, J., Benjamin, S., Madsen, B., Nocente, M., Rasmussen, J., \& Schmidt, B. S. (2021). Fast-ion orbit sensitivity of neutron emission spectroscopy diagnostics. Review of Scientific Instruments, 92(4), [043526]. https://doi.org/10.1063/5.0040696

\section{General rights}

Copyright and moral rights for the publications made accessible in the public portal are retained by the authors and/or other copyright owners and it is a condition of accessing publications that users recognise and abide by the legal requirements associated with these rights.

- Users may download and print one copy of any publication from the public portal for the purpose of private study or research.

- You may not further distribute the material or use it for any profit-making activity or commercial gain

- You may freely distribute the URL identifying the publication in the public portal

If you believe that this document breaches copyright please contact us providing details, and we will remove access to the work immediately and investigate your claim 


\section{Fast-ion Orbit Sensitivity of Neutron Emission Spectroscopy Diagnostics}

H. Järleblad, ${ }^{1,}$ a) L. Stagner, ${ }^{2}$ M. Salewski, ${ }^{1}$ J. Eriksson, ${ }^{3}$ S. Benjamin, ${ }^{4}$ B. Madsen, ${ }^{1}$ M. Nocente, ${ }^{5} \mathrm{~J}$. Rasmussen, ${ }^{1}$ and B. S. Schmidt ${ }^{1}$

1) Department of Physics, Technical University of Denmark, DK-2800 Kgs. Lyngby, Denmark

2) General Atomics, P.O. Box 85608, San Diego, California 92186-5608, USA

3) Department of Physics and Astronomy, Uppsala University, 75120 Uppsala, Sweden

${ }^{4)}$ Mathematical Sciences Institute, Australian National University, Canberra ACT 2601, Australia

${ }^{5)}$ Department of Physics, University of Milano-Bicocca, 20126 Milano, Italy

Fast ions in fusion plasmas often leave characteristic signatures in the plasma neutron emission. Measurements of this emission are subject to the phase-space sensitivity of the diagnostic, which can be mapped using weight functions. In this paper, we present orbit weight functions for the TOFOR and NE213 neutron diagnostics at the Joint European Torus (JET), mapping their phase-space sensitivity in 3D orbit space. Both diagnostics are highly sensitive to fast ions that spend a relatively large fraction of their orbit transit times inside the viewing cone of the diagnostic. For most neutron energies, TOFOR is found to be relatively sensitive to potato orbits and heavily localized counter-passing orbits, as well as trapped orbits whose 'banana tips' are inside the viewing cone of TOFOR. For the NE213 scintillator, the sensitivity is found to be relatively high for stagnation orbits.

\section{INTRODUCTION}

The age of burning plasmas is quickly approaching. With the ITER tokamak ${ }^{1,2}$ and smaller projects such as $\mathrm{SPARC}^{3}$, burning plasmas will pose novel challenges in regard to dominant alpha particle heating. Fast alpha particles are created in fusion reactions between fast deuterium (D) and tritium (T) ions, which will be further explored in the upcoming $\mathrm{DT}$ campaign at . . $\mathrm{JT}^{4}$ The behaviour of these fast ions may lead to undesired losses in energy and fast-ion density ${ }^{5}$ of varying severity, such as via the development of energetic particle modes ${ }^{6}$ via the interaction with Al 1 vén eigenmodes ${ }^{7-9}$ and via the interaction with sawteeth instabilities ${ }^{10-13}$. Understanding the behaviour and physics of these fast ions is therefore considered paramount. ${ }^{14-16}$ Prior to the development of the tools of velocity-space tomography ${ }^{17-23}$, an assessment of how the fast ions are distributed in velocity space was only possible by simulations of the fast-ion distribution function and finding the simulation matching the experimental data the best. ${ }^{24,25}$ With velocityspace tomography, a reconstruction of the fast-ion distribution from measurements became possible via weight functions ${ }^{14}$. A weight function $w$ is the phase-space sensitivity of a diagnostic. When its product with the fast-ion distribution $f$ is integrated over phase space $(\mathbf{x}, \mathbf{v})$, the diagnostic signal $s$ is obtained. This can be expressed as

$$
s\left(E_{1, d}, E_{2, d}\right)=\iint w\left(E_{1, d}, E_{2, d}, \mathbf{x}, \mathbf{v}\right) f(\mathbf{x}, \mathbf{v}) d \mathbf{x} d \mathbf{v}
$$

where $E_{1, d}, E_{2, d}$ are used to indicate the boundaries of an energy bin in which particles or photons are detected by the diagnostic. From here on, the dependence on $E_{1, d}, E_{2, d}$ will be omitted for brevity. For a single diagnostic energy bin, the weight functions used in velocityspace tomography are two-dimensional, with energy $(E)$

a)henrikj@dtu.dk and pitch $(p)$ dependence. Utilizing velocity-space weight functions with tomography, it is possible to infer the fast-ion distribution in a small measurement volume around a single cross-sectional point $(R, Z)$. Velocityspace weight functions have been developed for various fast-ion diagnostics, including fast-ion $\mathrm{D}-\alpha$ spectroscopy $(\text { FIDA })^{26,27}$, collective Thornson scattering ${ }^{28,29}$, gammaray spectroscopy ${ }^{30,31}$, fast ion loss detectors ${ }^{32}$, neutrat particle analyzers ${ }^{28}$, neutron emission spectroscopy $(\mathrm{NES})^{33,34}$ as well as 1D weight functions for ion cyclotron emission diagnostics ${ }^{35}$. However, with velocityspace tomography, the full 3D fast-ion distribution of all ions in the tokamak eludes reconstruction. Orbit weight functions solve this problem by using the physical correlation of the points along fast-ion orbits. This enables an inference of the complete fast-ion distribution (assuming toroidal symmetry, guiding-centre picture and neglecting collisions) in three-dimensional orbit space ${ }^{36}$. The distribution in orbit space can then be transformed into the corresponding distribution in $(E, p, R, Z)$. Processes for calculating orbit weight functions have been developed for neutron scintillators, NPA and FIDA. ${ }^{37}$ This paper concerns the development of orbit weight functions for NES diagnostics. At the JET tokamak, neutron spectroscopy is well-established ${ }^{38}$, velocity-space weight functions have alreadly been developed ${ }^{33,34}$ and it is used as a main diagnostic for fast-ion studies. ${ }^{38,39}$. The development of NES orbit weight functions will enable more detailed studies of fast ions in burning plasmas at JET in the upcoming DT campaign.

This paper is organized as follows. In section II, the choice of orbit coordinates is motivated, the coordinates are explained and orbit space is described. In section III, an overview of how to calculate orbit weights is presented and described in more detail for the TOFOR ${ }^{40}$ and ${ }_{\text {NNE213-scintillator }}^{41}$ at JET. In section IV, orbit weight functions for NES at JET are presented and visualized. Thereafter, a conclusion and outlook follow in section V. 


\section{ORBIT SPACE $\left(E, p_{m}, R_{m}\right)$}

There are several possible choices when it comes to labelling orbits in a tokanak plasma. ${ }^{42,43,44}$ The labelling can be done via a chosen set of coordinates. The usual constants of motion coordinates $\left(E, \mu, p_{\phi}\right)=$ (energy, magnetic moment, toroidal canonical angular momentum) do not uniquely identify orbits ${ }^{43}$. Therefore, to be able to work with a purely $3 \mathrm{D}$ phase space, we have chosen to work with the so-called orbit-space coordinates $\left(E, p_{m}, R_{m}\right)^{37}$. Here $E$ is the kinetic energy of the fast ion and $p_{m}$ is the pitch (ion velocity parallel to the magnetic field divided by total ion velocity magnitude) at the maximum major radius position $R_{m}$ of (the guiding-center of) the orbit. Given a tokamak equilibrium and geometry, an $\left(E, p_{m}, R_{m}\right)$ triplet uniquely labels any orbit $\left(Z_{m}\right.$ is implicitly fixed). Some example orbits are shown in Figure 1 , illustrating the concept of an $\left(E, p_{m}, R_{m}\right)$ triplet. All orbits and relevant orbit-space quantities in this paper are calculated for a deuterium ion.

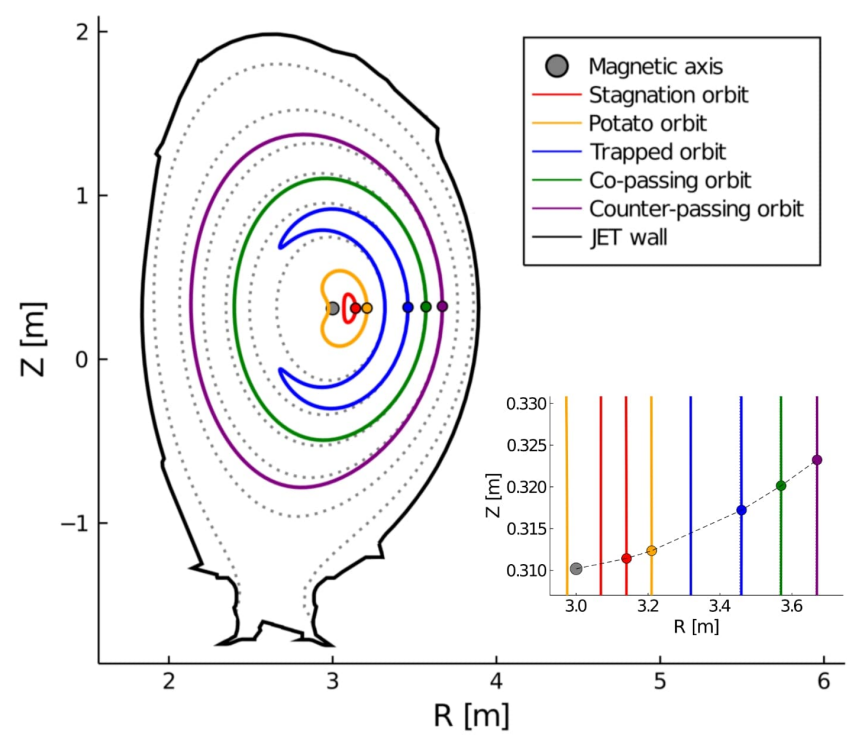

FIG. 1: Example orbits with $\left(E[\mathrm{keV}], p_{m}[-], R_{m}[\mathrm{~m}]\right)$ coordinates $(80,0.15,3.14)$ (stagnation), $(80,0.29,3.21)$ (potato), (80, 0.47, 3.46) (trapped), $(80,0.88,3.57)$ (copassing) and $(80,-0.88,3.67)$ (counter-passing) for JET shot \#96100 at 13.0012 seconds. The colored lines correspond to the trajectories of the fast-ion guiding center. The dotted lines correspond to the magnetic flux surfaces. The colored points mark the $R_{m}$-coordinates of the orbits. Notice how the $Z$-coordinates of the $R_{m^{-}}$ points are slightly increasing with $R_{m}$, as a result of the specific magnetic equilibrium.

In orbit space there are specific topological regions corresponding to the different types of orbits. In contrast to particle space $(E, p, R, Z)$, the gradient of the fast-ion distribution is usually not continuous across topological boundaries in orbit space. This is because continuity implies correlation, and it is not always the case that topological regions that are close together in orbit space are correlated. When crossing the boundary between two topological regions, the orbit trajectory can change dramatically. It is even possible that areas that are far apart in orbit space are more closely correlated than areas that are nar to each other ${ }^{5}$. From Figure 2, we can observe how a large portion of orbit space (gray region) corresponds to invalid and lost orbits. Lost orbits are orbits that intersect the tokamak wall or the divertor. Invalid orbits are orbits that are impossible to realize, given the magnetic equilibrium. The size of this gray region can vary between tokamaks, time-stamps etc. However, as previously investigated ${ }^{45}$ for the DIII-D tokamak, it seems to consistently extend over a large portion of orbit space. Slices through orbit space at other energies are qualitatively similar.

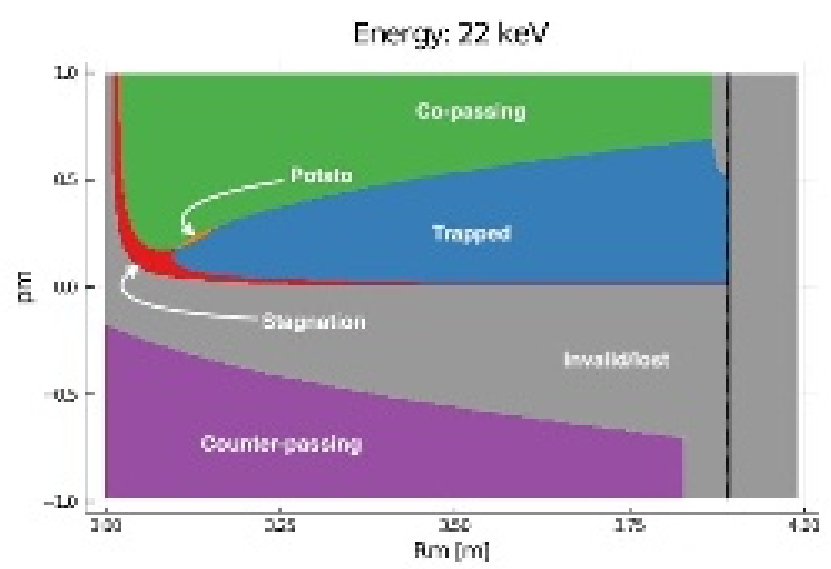

FIG. 2: Orbit-space topology for JET shot \#96100 at 13.0012 seconds with energy $E=22.0 \mathrm{keV}$ held constant. The black line marks the low-field side JET wall.

\section{CALCULATING ORBIT WEIGHTS}

To calculate a weight, one calculates the signal produced by an ion at a specific action coordinate $\mathbf{J}$, averaged over the angle coordinates $\boldsymbol{\Theta}$. It can be stated generally as ${ }^{45}$

$$
w(\mathbf{J})=\left(\prod_{i} \frac{1}{\tau_{i}}\right) \int_{0}^{\tau_{1}} \cdots \int_{0}^{\tau_{i}} S(\mathbf{J}, \boldsymbol{\Theta}) d \boldsymbol{\Theta}
$$

where $w$ is the weight, $\tau_{i}$ is the final value before the corresponding angle coordinate repeats and $S$ is the (synthetic) diagnostic signal. For our orbit space of interest, $\mathbf{J}=\left(E, p_{m}, R_{m}\right)$ and $\boldsymbol{\Theta}=\left(t, \gamma, \phi_{0}\right)$, where $t$ is the time, $\gamma$ is the gyro-angle and $\phi_{0}$ is the initial toroidal angle. Equation (2) can thus be written specifically as

$$
w=\frac{1}{4 \pi^{2} \tau_{p}} \int_{0}^{2 \pi} \int_{0}^{2 \pi} \int_{0}^{\tau_{p}} S\left(E, p_{m}, R_{m}, t, \gamma, \phi_{0}\right) d t d \gamma d \phi_{0}
$$

where $w=w\left(E, p_{m}, R_{m}\right)$ is the orbit weight for a single $\left(E, p_{m}, R_{m}\right)$ coordinate. The orbit weights are the signal 


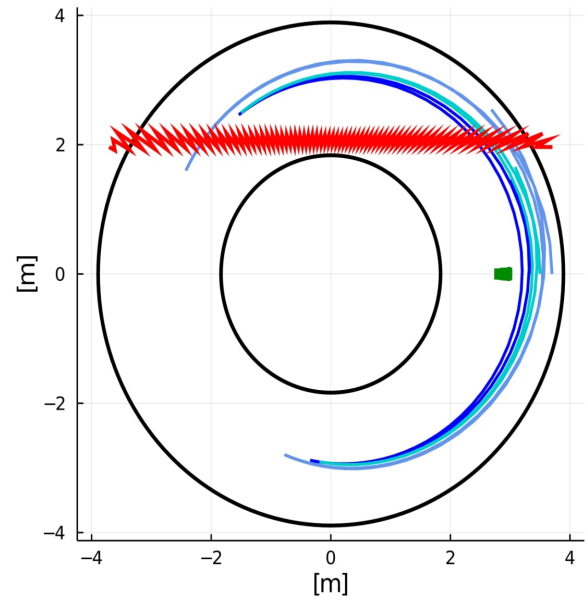

a)

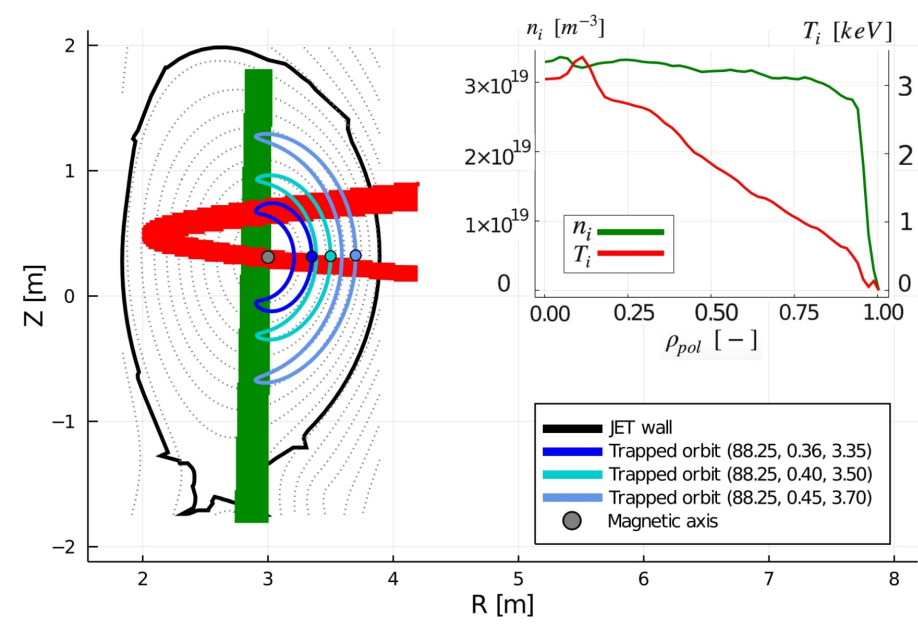

b)

FIG. 3: Viewing cones of the TOFOR and NE213 diagnostics are shown by green and red areas, respectively. Depicted as examples in a) and b), both diagnostics observe part of the fast-ion guiding-center trajectories for the trapped orbits with shown $\left(E[\mathrm{keV}], p_{m}[-], R_{m}[\mathrm{~m}]\right)$ coordinates. The equilibrium is th $\$$ ame as in Figure 1 . The ion temperature and density profiles are shown in b) as subfigure.

per fast ion on that orbit. In practice, the orbit weight functions are then calculatt by discretizing (3), calculating synthetic signals via a forward model for distributions consisting of just one non-zero orbit-space voxel (a representation of a delta function), averaging over $\left(t, \gamma, \phi_{0}\right)$ and structuring the signals into a matrix $W$. Calculating $W$ can be written as the following step-by-step process.

1. Let $f=n_{f} \delta\left(E-E_{i}\right) \delta\left(p_{m}-p_{m, j}\right) \delta\left(R_{m}-R_{m, k}\right)$ where $f$ is the test fast-ion distribution and $n_{f}$ is a test fast-ion density

2. Transform $f$ into the format needed by the forward model as input

3. Use the forward model to calculate the signal $S$ for that delta function

4. Average over $\left(t, \gamma, \phi_{0}\right)$

5. Repeat for all $\left(E_{i}, p_{m, j}, R_{m, k}\right)$ points of interest.

In the case of NES, we are interested in how different orbits produce different neutron spectra. Or equivalently, how sensitive a measurement at a specific neutron energy is to different areas of orbit space. We have focused on the TOFOR ${ }^{40}$ diagnostic and the NE213-scintillator ${ }^{41}$ diagnostic, both installed at JET. TOFOR is installed so as to view a collimated neutron flux coming up vertically from the plasma. As can he seen in Figure 3, TOFOR and NE213 are able to observe neutrons originating from fusion reactions along the part of the orbit that lies in the viewing cone of the diagnostic. Depending on the orbit, the fast ion will spend a varying amount of time inside the viewing cone. For both diagnostics (or any diagnostic), this will result in different parts of orbit space acquiring different weights. The neutron energy $E_{n}$ can be related to the known motional state of the reactants via the flollowing equation ${ }^{3946}$.

$$
\begin{aligned}
E_{n} & =\frac{1}{2} m_{n} V_{c m}^{2}+\frac{m_{n}}{m_{n}+m_{f}}(Q+K) \\
& +\left(V_{c m} \cos \theta\right)\left(\frac{2 m_{n} m_{f}}{m_{n}+m_{f}}(Q+K)\right)^{1 / 2} .
\end{aligned}
$$

Here $m_{n}$ is the neutron mass and $V_{c m}=\left|\mathbf{V}_{\mathbf{c m}}\right|$ where $\mathbf{V}_{\mathbf{c m}}=\left(m_{1} \mathbf{v}_{\mathbf{1}}+m_{2} \mathbf{v}_{\mathbf{2}}\right) /\left(m_{1}+m_{2}\right)$ is the center-ofmass velocity of the two reactant ions, described by their masses $m_{1}$ and $m_{2}$ and velocities $\mathbf{v}_{\mathbf{1}}$ and $\mathbf{v}_{\mathbf{2}} \cdot m_{f}$ is the mass of the second product of the fusion reaction, $Q$ is the nuclear energy release of the reaction, $K=m_{1} m_{2} v_{r e l}^{2} /\left(2\left(m_{1}+m_{2}\right)\right)$ is the relative kinetic energy of the reactants where $v_{r e l}=\left|\mathbf{v}_{\mathbf{2}}-\mathbf{v}_{\mathbf{1}}\right|$ and $\theta$ indicates the angle between $\mathbf{V}_{\mathbf{c m}}$ and the neutron velocity vector in the center-of-mass frame. In practice, the process of calculating an NES orbit weight (for a given plasma equilibrium and a given bulk ion temperature profile and density profile) is as follows. An orbit is calculated for an $\left(E, p_{m}, R_{m}\right)$ triplet. Toroidal symmetry is assumed, which means that the orbit is characterized by its projection onto the $(R, Z)$ plane and by the energy and pitch values at each $(R, Z)$ point. Hence, this is effectively an $(E, p, R, Z)$ distribution representing the orbit. For a given instrument (TOFOR or NE213), the expected diagnostic signal from this distribution can then be calculated with Mbnte-Carlo methods ${ }^{47}$. We normalize these calculations to the total number of points on the orbit which gives the signal that one ion on this orbit would give rise to, which is precisely the orbit weight that we are after. The orbit weight functions presented in this work have been calculated for a $100 \times 100 \times 100$ orbitspace grid with $E:[20.65,149.35] \mathrm{keV}, p_{m}:[-0.99,0.99]$ 
and $R_{m}:[3.03,3.80] \mathrm{m}$. To achieve these relatively smooth weight functions, no more than $500(E, p, R, Z)$ points were used for each orbit. Often the number of $(E, p, R, Z)$ points was significantly lower. The computational time is dependent on a number of factors. To calculate the orbit weight functions presented in this paper, 40 Intel(R) Xeon(R) Scalable Gold CPUs 61482.20 $\mathrm{GHz}$ were used, with an estimated maximum of $17.21 \mathrm{~GB}$ utlized RAM memory and a total computational time of approximately 25 hours (including programming package initialization and calculation of the orbits). However, it should be noted that an orbit weight function calculated on a 100x100x100 orbit-space grid uses about five orders of magnitude more valid orbits than what is needed to accurately mimic the physics of the forward model in this case. For JET shot \#96100, an orbit weight function using just the valid orbits of a $17 \times 15 \times 13$ orbitspace grid (for example) is enough to closely reproduce the expected diagnostics signal, when multiplied with the fast-ion dist iibution (equation (1)). The number of valid orbits needed to correctly mimic the physics of the forward model with the orbit weight functions will depend on the size of the non-zero areas of the fast-ion distribution in phase space as well as the phase-space resolution requirements for both the fast-ion distribution and the diagnostic.

\section{VISUALISATION OF ORBIT WEIGHT FUNCTIONS}

Since orbit weight functions are three-dimensional, the process of visualisation is difficult. For every point in three-dimensional orbit space there is a corresponding weight. Thus the data is four-dimensional (one phasespace location and one weight). Previous work has visualised orbit weight functions via projection onto the three orthogona eoordinate planes. ${ }^{37}$ To be able to get a detailed picture of how the sensitivity is distributed in terms of different orbit types, this work has chosen to instead examine the orbit weight functions slice-by-slice in terms of fast-ion energy $E$. The topological boundaries for a specific energy can then be superimposed onto the orbit weight function to easily identify the topological areas of highest sensitivity. In Figure 4, it can be observed how, for the given fast-ion energy and TOFOR neutron energy bin, large weights can be found for potato orbits and heavily localized counter-passing orbits. It can also be noted how a narrow region of large weights extends from the potato region, through the trapped region and out to the JET wall. This is due to the 'banana tips' of those trapped orbits coinciding with the TOFOR viewing cone, where the fast ions then spend a relatively large fraction of their poloidal transit times. Hence, the sensitivity is higher. For the NE213-scintillator, the general structure of the orbit weight functions differs, as can Be seen in Figure 5. For the given fast-ion energy and neutron energy bin, the sensitivity to stagnation orbits is relatively high. This is in agreement with expectations,

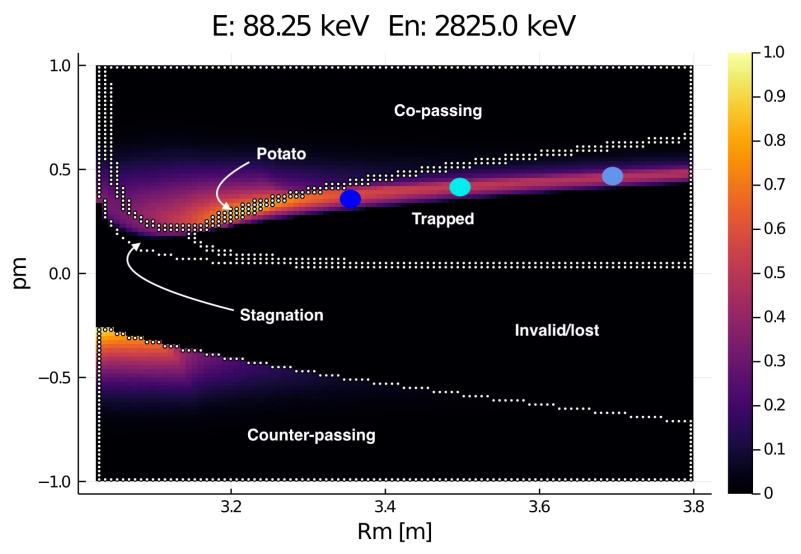

FIG. 4: A slice of a normalized TOFOR orbit weight function with superimposed topological boundaries. For a fast-ion orbit with $E=88.25 \mathrm{keV}$, the TOFOR neutron energy bin with

$\left(E_{1, d}, E_{2, d}\right)=(2.775,2.875) \mathrm{MeV}$ will be most sensitive to potato orbits and counter-passing orbits heavily localized around the magnetic axis (small $R_{m}$ value). The three colored points indicate the coordinates of the trapped orbits depicted in Figure 3 a) and b). The equilibrium is the same as in Figure 1. The colorbars indicate normalized weight.

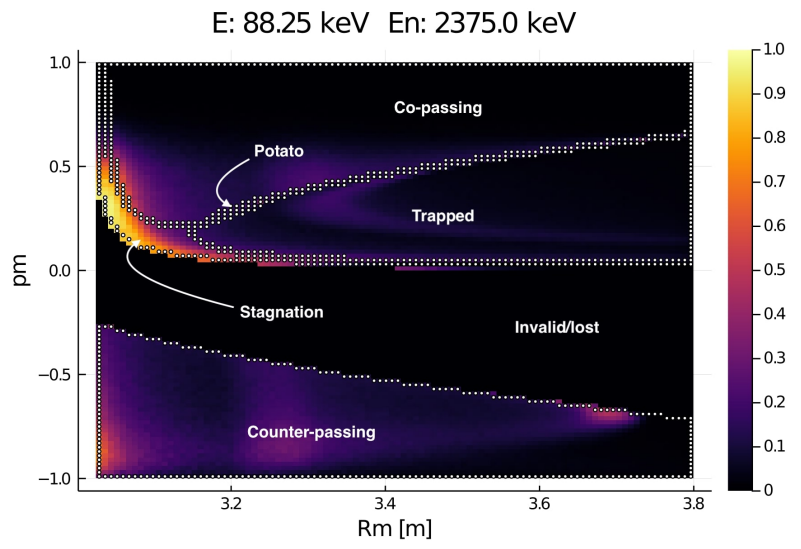

FIG. 5: A slice of a normalized NE213 orbit weight function with superimposed topological boundaries. For a fast-ion orbit with $E=88.25 \mathrm{keV}$, the NE213 neutron energy bin with $\left(E_{1, d}, E_{2, d}\right)=(2.325,2.425) \mathrm{MeV}$ will be most sensitive to stagnation orbits. The equilibrium is the ame as in Figure 1. The colorbars indicate normalized weight.

since many stagnation orbits will have their poloidal orbit path projection completely within the NE213 viewing cone. The slices presented in Figure 4 and Figure 5 are typical for most neutron energy bins and fast-ion energies, albeit great variations do exist. Note that the above results have been obtained assuming DD reactions only and exclusively beam-target neutron yield. The orbitspace sensitivity will likely vary for other scenarios. Re- 
call also that the topology of orbit space itself will vary depending on the magnetic equilibrium. However, the discussion regarding orbits with a relatively high sensitivity attributable to a relatively large fraction of the time being spent within the viewing cone of the diagnostic is valid in general.

\section{CONCLUSION AND OUTLOOK}

Orbit weight functions have been developed for the TOFOR and NE213-scintillator diagnostics at JET. They are comprised of weights where each weight is the signal per fast ion on a unique orbit. On their own, they describe the orbit-space sensitivity. That is, for a given diagnostic, how sensitive a measurement of a specific energy bin is to the different regions of orbit space. The weight functions show that both diagnostics are highly sensitive to orbits whose fast ions spend a large fraction of their orbit transit times inside the viewing cone of the diagnostic. In particular, TOFOR is found to have relatively large weights for potato orbits and heavily localized counter-passing orbits. The NE213-scintillator is found to have relatively large weights for stagnation orbits. Together with a TOFOR- or NE213-signal originating from a fast-ion distribution, the orbit weight functions can be used to reconstruct the orbit-space fast-ion distribution, which can then be transformed to obtain the four-dimensional, gyro- and toroidally-averaged fastion distribution function in energy, pitch, $\mathrm{R}$ and $\mathrm{Z}$. This will be shown in future work.

\section{DATA AVAILABILITY STATEMENT}

Raw data were generated at the JET large scale facility. Derived data supporting the findings of this study are available from the corresponding author upon reasonable request.

\section{ACKNOWLEDGMENTS}

We thank the ITPA Energetic Particle Physics Topical Group for its support. This work has been earrice out within the framework of the EUROfusion Consortium and has received funding from the Euratom research and training programme 2014-2018 and 2019-2020 under grant agreement No 633053. The views and opinions expressed herein do not necessarily reflect those of the European Commission.

${ }^{1}$ M. Salewski et al, Nucl. Fusion 58, 096019 (2018).
${ }^{2}$ B. Bigot, Nucl. Fusion 59, 112001 (2019).

${ }^{3} \mathrm{P}$. Rodriguez-Fernandez et al, Journal of Plasma Physics 86, 865860503 (2020).

${ }^{4}$ E. Joffrin et al, Nucl. Fusion 59, 112021 (2019).

${ }^{5}$ M. Salewski et al, Nuclear Fusion 51, 083014 (2011).

${ }^{\circ} \mathrm{S}$. Briguglio et al, Physics of Plasmas 14 (2007), $10.1063 / 1.2710208$.

${ }^{7}$ W. W. Heidbrink, Physics of Flasmas 9, 2113 (2002).

${ }^{8}$ W. W. Heidbrink, Physics of Plas nas 15, 055501 (2008).

${ }^{9}$ B. Madsen et al, Nucl. Fusion 60, 066024 (2020)

${ }^{10}$ F.B. Marcus et al, Nucl. Fusion 34, 687 (1994).

${ }^{11}$ J. P. Graves et al, Plasma Phys. Control. Fusion 47, B121 (2005).

${ }^{12}$ D.J. Campbell et al, Phys. Rev. Letters 60 (1988).

${ }^{13}$ Ya. [. Kolesnichenko et al, Nucl. Fusion 40, 1325 (2000).

${ }^{14}$ M. Salewski, Fast-ion diagnostic in fusion plasmas by velocityspace tomography (Technical University of Denmark, Kongens Lyngby, Denmark, March 2019).

${ }^{15}$ M. Salewski et al, Journal of Instrumentation 14, C05019 (2019).

${ }^{10} \mathrm{~J}$. Eriksson et al, Nuclear Fusion 55, 123026 (2015).

${ }^{17}$ M. Salewski et al, Nucl. Fusion 54, 023005 (2014).

${ }^{18}$ A.S. Jacobsen et al, Plasma Phys. Cont. (2016).

${ }^{19} \mathrm{M}$. Weiland et al, Plasma Phys. Contrpl. Fusion 58, 025012 (2016).

${ }^{20}$ M. Weiland et al, Nucl. Fusion 57, 116058 (2017)

${ }^{21}$ M. Salewski et al, Nucl. Fusion 57, 056001 (2017).

${ }^{22}$ B. Madsen et al, Nucl. Fusion 60, 066024 (2020).

${ }^{23}$ B. Madsen et al, Plasma Phys. Control. Fusion 62, 115019 (2020).

${ }^{24}$ M. Salewski et al, Nucl. Fusion 50, 035012 (2010).

${ }^{25}$ M. Salewski et al, Nucl. Fusion 53, 063019 (2013).

${ }^{26}$ M. Salewski et al, Plasma Phys. Contrbl. Fusion 56, 105005 (2014).

${ }^{27} \mathrm{~W}$. W. Heidbrink et al, Plasma Phys. Control. Fusion 49, 1457 (2007).

${ }^{28}$ M. Salewski et al, Fusion Science and Technology 74, 23 (2018).

${ }^{29}$ M. Salewski et al, Nucl. Fusion 51, 083014 (2011).

${ }^{30}$ M. Salewski et al, Nucl. Fusion 55, 093029 (2015).

${ }^{31}$ M. Salewski et al, Nucl. Fu;ion 56, 046009 (2016).

${ }^{32} \mathrm{~J}$. Galdon-Quiroga et al, Plasma Phys Control. Fusion 60, 105005 (2018).

3 A. S. Jacobsen et al, Nucl. Fusion 55, 053013 (2015).

${ }^{4}$ A. S. Jacobsen et al, Rev. Sci. Instrum. 88, 073506 (2017).

${ }^{35}$ B. S. Schmidt et al, "Determining 1D Fast-Ion Velocity Distribution Functions from Ion Cyclotron Emission Data Using Deep Neural Networks," These proceedings.

${ }^{36}$ L. Stagner, "Orbit Tomography of Energetic Particle Distribution Functions," Submitted.

${ }^{37}$ L. Stagner and W. W. Heidbrink, Physics of Plasmas 24, 092505 (2017).

${ }^{38}$ J. Eriksson et al, Plasma Phys. Control. Fusion 61, 014027 (2019).

${ }^{39}$ D. Moseev et al, Rev. Mod. Plissma Phys. 2, 7 (2018)

${ }^{40} \mathrm{M}$ Gatu Johnson et al, Nucl. Instrum. ]Methods Phys. Res. A 591, 417 (2008).

${ }^{41}$ F. Binda et al, Rev. Sci. Inst.um. 85, 11E23 (2014).

${ }^{42}$ R. B. White, The Theory of Toroidally Confined Plasmas (Imperial College Press, 2nd Edition, 2001).

43 JA Rpme and YK M. Peng, Nuclear Fusion 19, 1193 (1979).

${ }^{44} \mathrm{Yu} V$ Perov and RW Harvey, Plasma F'hysics and Controlled Fusion 58, 115001 (2016).

${ }^{45} \mathrm{~L}$. Stagner, Inference of the Fast-ion Distribution Function, Ph.D. thesis, University of California, Irvine (2018).

${ }^{46}$ H. Brysk, Plasma Phys. 15, 611 (1973).

${ }^{47} \mathrm{~J}$. Eriksson et al, CPC 199, 40 (2016). 


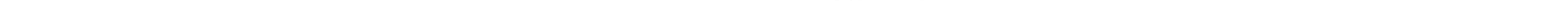


Energy: $22 \mathrm{keV}$

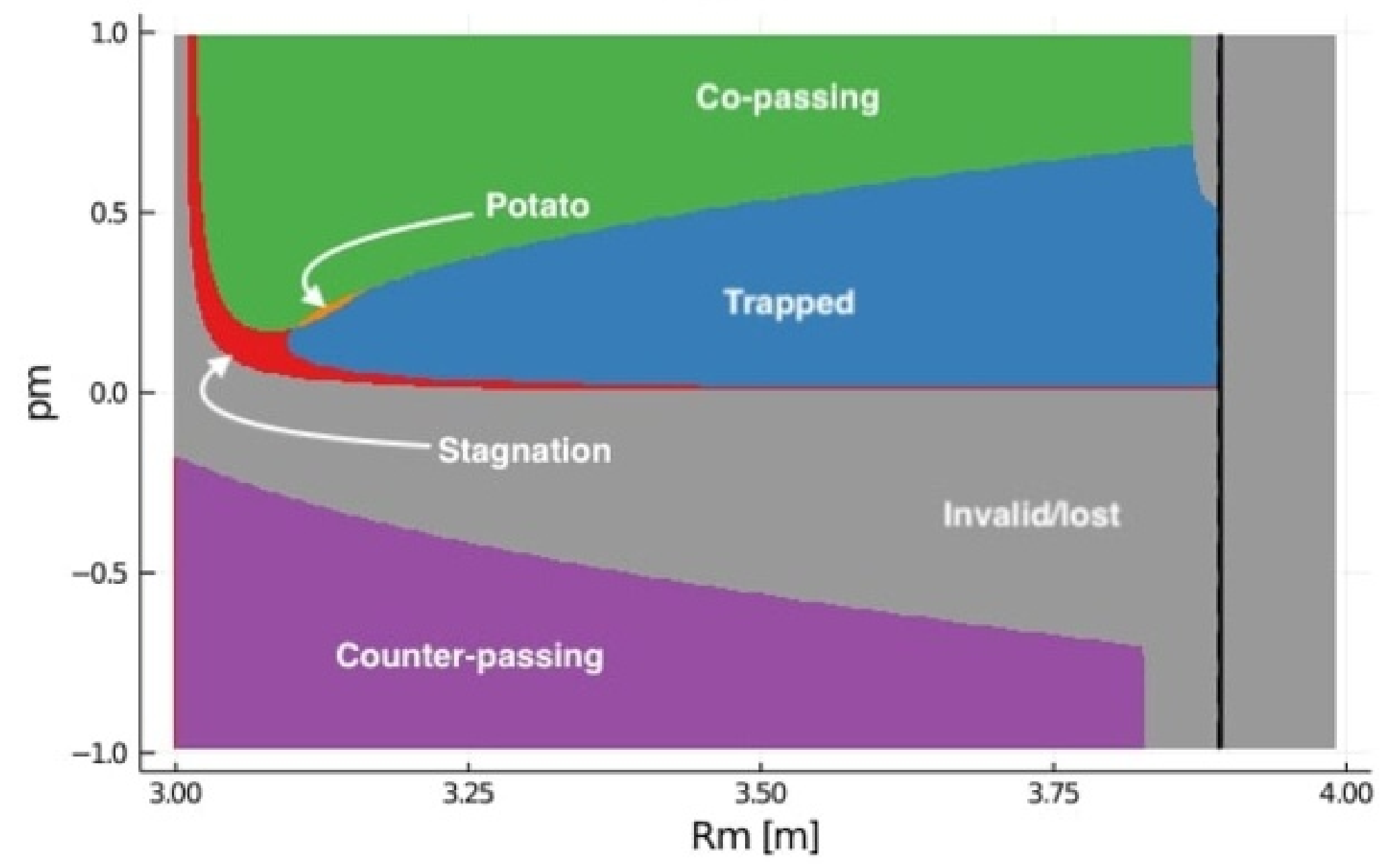




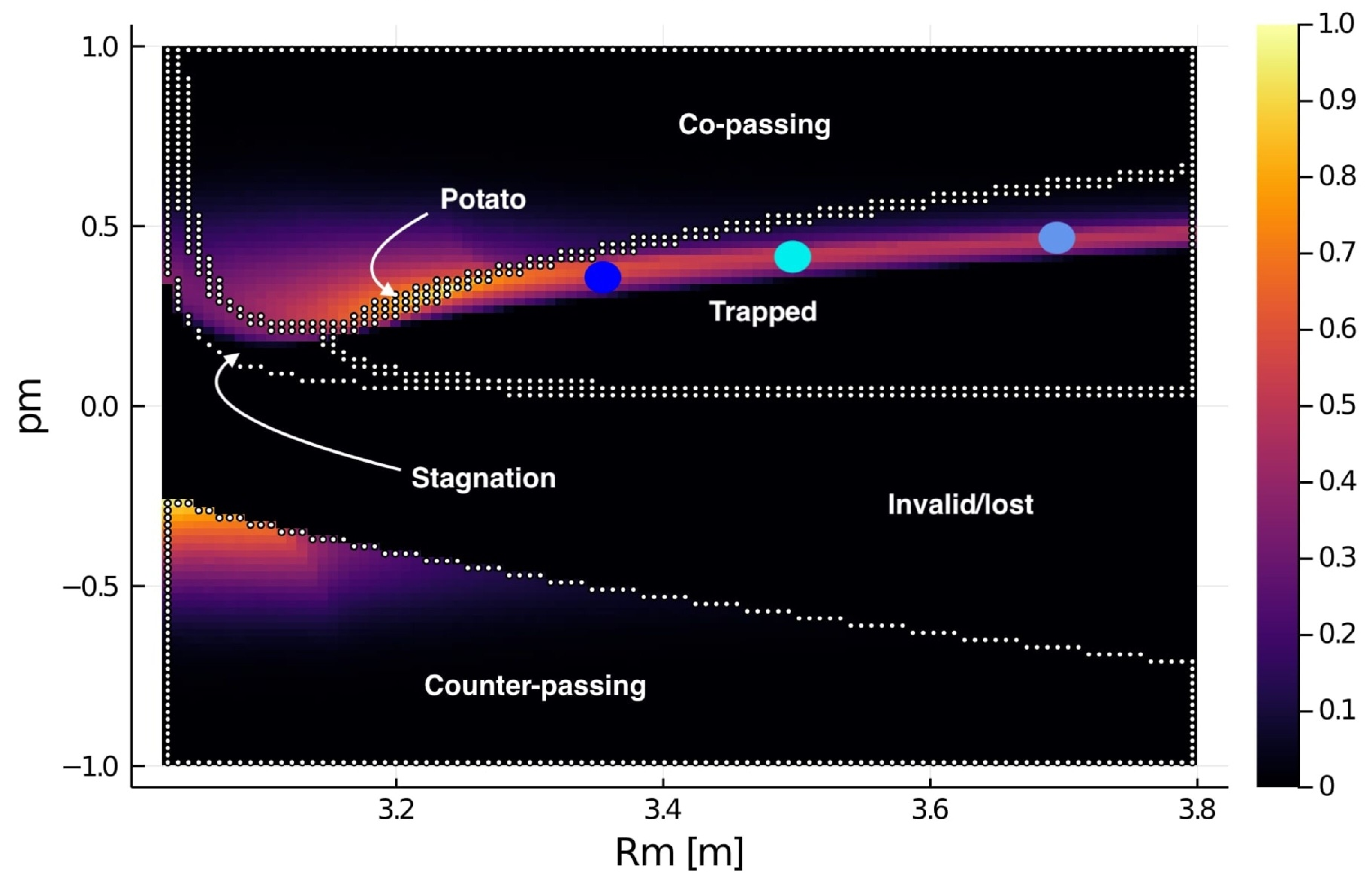




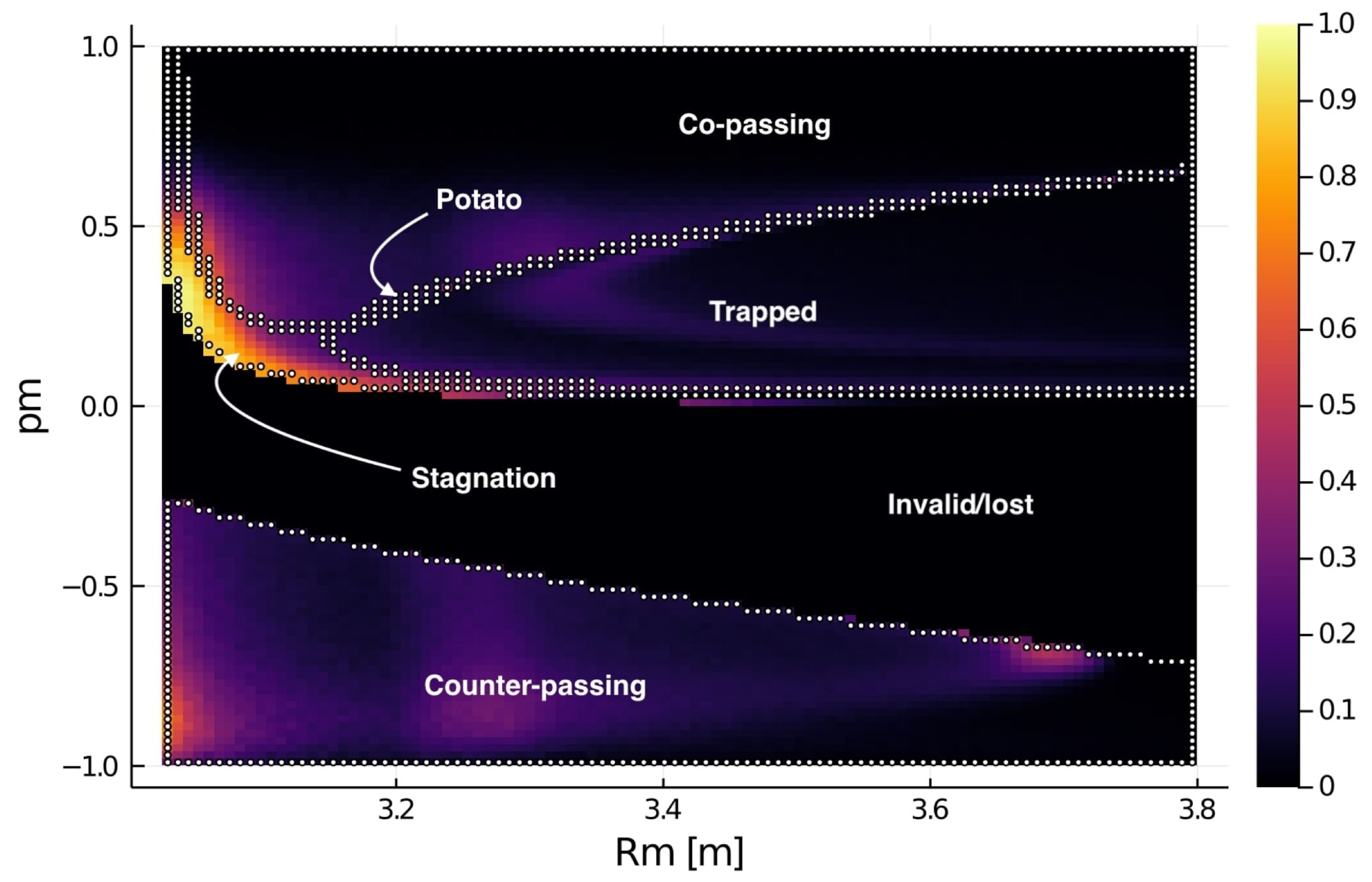

